Research Article

\title{
Practice of Programming Education using Finger Robot
}

\author{
Kaito Omata ${ }^{1}$, Shinichi Imai ${ }^{2 * *}$ \\ ${ }^{1}$ Graduate School of Education, The University of Tokyo, 7-3-1 Hongo, Bunkyo-ku, Tokyo 113-0033, Japan \\ ${ }^{2}$ Graduate School, Tokyo Gakugei University, 4-1-1 Nukuikita-machi, Koganei, Tokyo 184-8501, Japan
}

\author{
ARTICLE INFO \\ Article History \\ Received 08 September 2018 \\ Accepted 17 November 2018 \\ Keywords \\ Programming education \\ binary number \\ robot \\ CS unplugged
}

\begin{abstract}
The movement of teaching computer science to children can be seen all over the world, but because it contains abstract content, it is difficult for children like elementary school students to understand it through lectures alone. The purpose of this study is to develop teaching materials that stimulate interest in the concept of binary numbers by using robots to teach computer science. As a result of having children use the developed material, it helped them understand binary numbers.
\end{abstract}

(c) 2020 The Authors. Published by Atlantis Press SARL. This is an open access article distributed under the CC BY-NC 4.0 license (http://creativecommons.org/licenses/by-nc/4.0/).

\section{INTRODUCTION}

Today is the fourth industrial revolution. In Japan, the national curriculum was revised in 2017, and the new national curriculum will be implemented in elementary schools from FY 2020. The new national curriculum will require programming education at the elementary school level. Turning to the rest of the world, from 2014 England made "Computing" a compulsory subject at the primary school level [1]. From a global perspective, it can be seen that the knowledge, skills, qualities and abilities of computers and programming are considered to be necessary in the future.

Toward 2020, lessons using visual programming languages and lessons using robots are being tried as practical programming education in various parts of Japan [2,3]. It has been reported that, according to the lessons using robots, robots can attract children's interest even if they do not have a high level of interest and understanding of scientific concepts, are good at carrying out thinking try and error, and can work on programming while having fun.

Most of the robots used in programming lessons are mobile robots. The mobile robot has the advantage of being able to observe the behavior of the robot at the time of program execution, and being able to understand the control structure and its necessity step-by-step by case of repetition and collision. However, there are few teachers who can teach robot programming and it is difficult for children to understand and program sensors and motors. Development of robot teaching materials that are simple in programming and that operate without a deep understanding of sensors and motors facilitates robot programming that has many advantages in programming education.

"Corresponding author.Email: shimai@u-gakugei.ac.jp
An approach to teaching computer science to elementary school children is "COMPUTER SCIENCE Unplugged" (Next, CS Unplugged), developed by Bell et al. [4] in New Zealand. CS Unplugged is a simple way for elementary school students to learn the basic principles of computers. Examples include binary numbers, image representations, and text compression. In the computer world, information is represented in binary. In the process of learning programming, learning how a computer works will help you better understands computers and programming.

Learning materials that teach programming and the basic principles of computers at the same time will be needed in the future. In this research, we develop a robot teaching material that can simultaneously learn binary numbers and programming.

\section{EDUCATIONAL ROBOT TEACHING MATERIALS}

The proposed teaching material is a robot (Next, "Finger Robot") which imitates the right hand of a human as shown in Figure 1, and consists of the back of the hand and five fingers. The Finger Robot uses the robot kit of Artic Co., Ltd (Osaka, Japan). Studuino is used as a controller to operate the Finger Robot. The software uses Studuino Software, a block programming language, so that even elementary school students can easily handle programming.

\subsection{Hardware}

Studuino is connected to a Personal Computer (PC) via a USB cable. A servomotor is used as a finger moving mechanism. An external power supply (dry battery) is used as a power source to move the servomotor. Studuino can be used in two patterns: communication 
mode and transfer mode. The communication mode is to use Studuino and PC connected all the time, and the communication can be carried out all the time. The transfer mode is to write a program to Studuino's RAM and it can also be used by Studuino alone. In this teaching material, it is used in the communication mode in order to display the state of the Finger Robot on the screen of PC.

A Finger Robot can be operated by using a servomotor for each finger and connecting a terminal to the I/O port of Studuino. Unlike a normal motor, a servomotor can be positioned according to a specified angle. Studuino Software is able to run the block shown in Figure 2 simply by specifying an angle. A servomotor of $90^{\circ}$ is in a state where the fingers are stretched as shown in Figure 1. The servomotor is bent at $180^{\circ}$.

\subsection{Software}

When a robot is moved by programming, it is possible to move a finger without using conditional branching or manipulation of variables. Since the purpose of this educational material is to arouse interest in the concept of binary numbers, the program shown in Figure $3 \mathrm{a}$ is prepared in advance as a function so that the child can operate the servomotor by executing the block shown in Figure $3 \mathrm{~b}$. Although English is used in the figure, it is used by children in Japanese.

Figure $3 \mathrm{~b}$ is a function for operating the servomotor according to the state of the finger portion. The condition specified in the argument is a variable representing the state of the finger part, and in Figure $3 \mathrm{a}$, if the function is called when the finger part representing position 16 is extended, the finger part is bent, and if it is called when it is bent, it is stretched. Five functions, one for each finger, were prepared as shown in Figure 3b.

In the activity in the lesson, the child makes the program which moves the finger part using the function, and experiences the conversion between 10 and binary numbers based on the idea of the bit string, while bending and stretching the finger. The programs prepared by children are a combination of those shown in Figure 3b. As an example, in the case where all the finger portions are

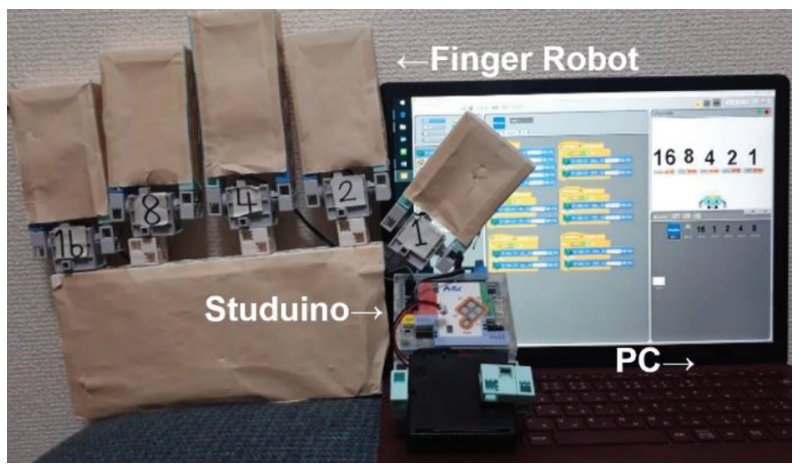

Figure $1 \mid$ Finger Robot.

Figure $2 \mid$ Block that specifies the angle.

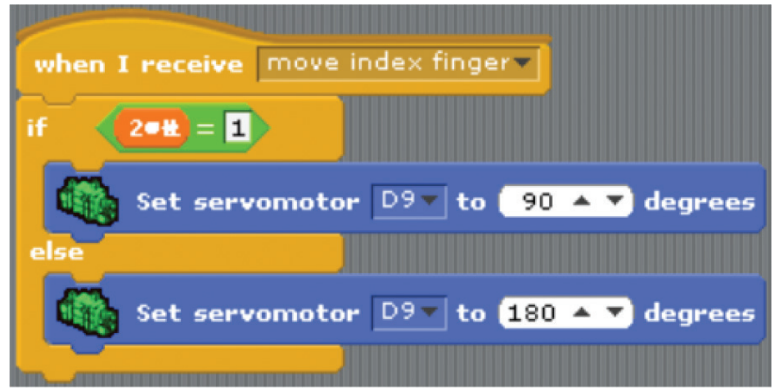

(a)

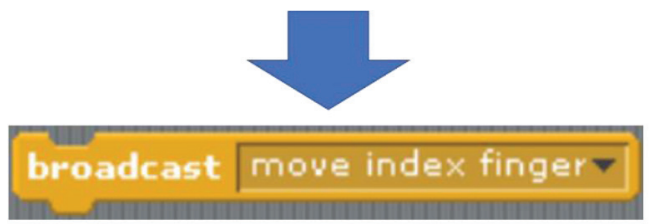

(b)

Figure 3 Function to operate the servomotor. (a) Program before functioning. (b) Functionalized blocks.

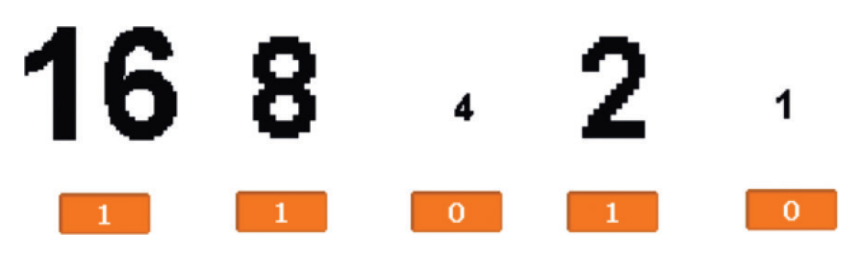

Figure $4 \mid$ Screen of the stage.

extended as shown in Figure 1 to a state representing the binary number "10101", among the blocks shown in Figure 3b, the blocks relating to the ring finger and the index finger are combined.

During programming, a screen as shown in Figure 4 is displayed on the PC screen. As an example, the numbers displayed on the stage shown in Figure 4 indicate that " 4 " and " 1 " are smaller, and that the fingers corresponding to " 4 " and " 1 " are curved in the Finger Robot. And, the value which made the description of the finger to correspond to the binary number is displayed under the numeral, and " 0 " is displayed under " 4 " and " 1 " of Figure 4, and it is proven that " 11010 " is obtained when the state of the finger of Figure 4 is expressed in the binary number. In the case of Figure 4, children can visually confirm that the binary "11010" corresponds to the decimal 26 by adding up the increasing decimal number.

\subsection{Activity}

In lesson, children use Finger Robots in pairs. The teacher presents a problem to move the Finger Robot to a specified state. The questions will be asked in four different ways. The four problems are the shape of the finger as a photograph, the shape of the finger as a letter, the shape of the finger as a binary number, and the shape of the finger as a decimal number. For these problems, consider the program in pairs. The child checks the current state of the robot and then programs it to figure out which finger to move to get it into the state described in the problem. Run the program once and transform it from the current state to the problem state. After you run the program, make sure it is 
the same as the problem, fix the program, or work on the next problem. It is instructed not to change the present state every time a child makes a mistake.

\section{RESULTS OF USING TEACHING MATERIALS}

In order to evaluate the proposed teaching materials, we conducted a programming experience at the University Festival of Tokyo Gakugei University. Figure 5 shows the author performing a bit string experience for each child using the proposed teaching materials. The content of the experience is the content which solves the problem shown in Table 1 using the proposed teaching material. After the experience, the questionnaire on the teaching material was carried out for the child. The subjects were 13 children from 1 st grade to 5 th grade.

The questionnaire was prepared by five methods on eight questions including the items shown in Table 2 such as impression using this teaching material and idea on robot teaching material. From the result of the questionnaire, the children who answered "I want to take a programming class using this robot/I really want to take it" to Q6 were 92\%, and the children who answered "I think you understand the way of thinking about bit strings." to Q9 were 75\%. From this fact, it was proven that the child was interested in the idea of the bit string.

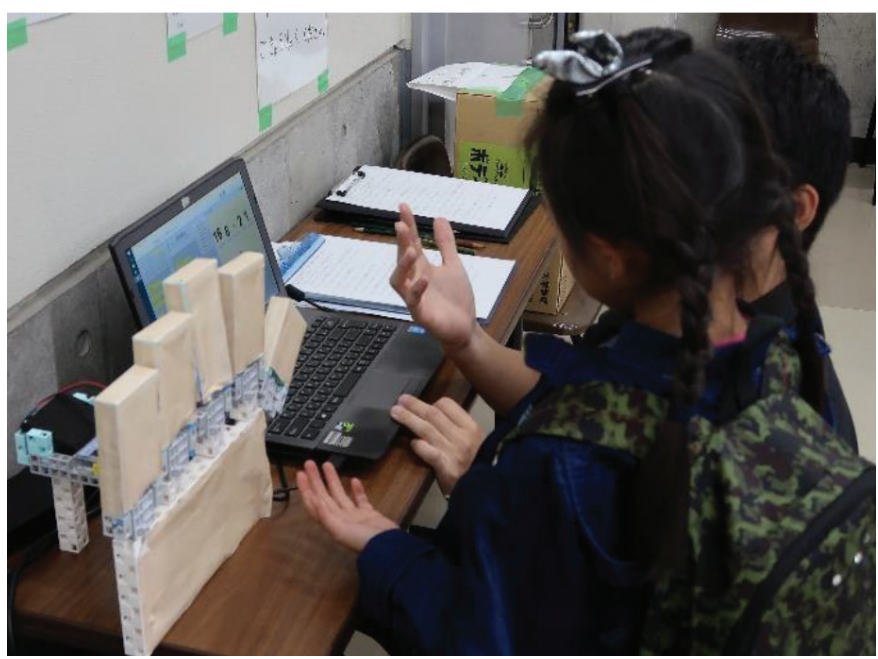

Figure 5 Scene of actual practice.
Table $1 \mid$ Problems solved by the children

\begin{tabular}{l} 
Number Problem statement \\
\hline (1) Shape fingers into a picture (In this article, the photograph is \\
omitted, but only the index finger and middle finger are bent.) \\
(2) Shape fingers like this "only the thumb and ring and little \\
fingers are bent" \\
(3) Shape fingers in the form of the binary number " 01010 " \\
(4) Shape fingers as a number " 26 "
\end{tabular}

Table $2 \mid$ Questions (Partial except)

\begin{tabular}{ll}
\hline Number & Questions \\
\hline Q6 & I want to take a programming class using this robot. \\
Q9 & If you take a class using this robot, you will understand \\
& well about "binary number" \\
\hline
\end{tabular}

\section{CONCLUSION}

In this research, developed a teaching material called Finger Robot that can be used for programming education in elementary schools. Using a Finger Robot, elementary school students can learn the idea of counting binary numbers with their fingers while experiencing programming. By associating the state of the Finger Robot with the binary representation and decimal number, children can deepen their understanding of the correspondence between binary and decimal numbers. In the future, plan to carry out endurance tests assuming the use of children, and to establish issues to deal with various developmental stages.

\section{CONFLICTS OF INTEREST}

The authors declare they have no conflicts of interest.

\section{REFERENCES}

[1] QCA, National curriculum in England: computing programmes of study, GOV.UK Department for Education, Manchester, 2013.

[2] K. Hagiwara, Robot programming for elementary school children, Mie Univ. Fac. Educ. Depart. Bull. Paper 68 (2017), 307-315.

[3] K. Kai, Y. Kimuro, Y. Sakaguchi, H. Yasuura, An educational method of computer principles for elementary and junior high school students. IPSJ, Transactions of Information Processing Society of Japan, 2002, pp. 1121-1131.

[4] T. Bell, I.H. Witten, M. Fellows, Computer science unplugged, Canterbury, 2015.

[5] F. Pohl, The best of Frederik Pohl, Ballantine Books Co., Ltd., London, 1975.

\section{AUTHORS INTRODUCTION}

\section{Mr. Kaito Omata}

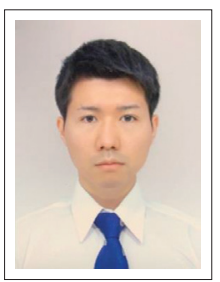

He received the Bachelor of Education in 2019 from Tokyo Gakugei University, Japan. Currently, he is a master course student at the University of Tokyo, Japan. His research interests include Programming Education and Psychology of Learning and Instruction.

\section{Dr. Shinichi Imai}

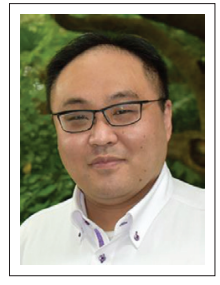

He graduated doctor course at department of engineering in Hiroshima University. He works at department of education in Tokyo Gakugei University. His research area is about control system design and educational engineering. 